\title{
Pulmonary Vesicle and Bullae Formation in Patients With COVID-19: Experiences from Wuhan
}

Hexiao Tang ${ }^{1 \&}, \mathrm{Li}_{\text {Zhang }}^{2 \&}$, Yanhong Wei ${ }^{3}$, Changsheng $\mathrm{Li}^{1}$, Bo Hu${ }^{4}$, Jinping Zhao ${ }^{1}$, Nahush A. Mokadam ${ }^{5}$, Hua Zhu ${ }^{5}$, Jun Lin ${ }^{6 *}$, Sufang Tian ${ }^{7 *}$, Xuefeng Zhou ${ }^{\text {* }}$

Nahush A. Mokadam, MD

Degree information for the other co-authors is $\mathrm{MD}, \mathrm{Ph} . \mathrm{D}$

${ }^{\&}$ Co-first author

${ }^{1}$ Department of Thoracic Surgery, Zhongnan Hospital of Wuhan University, Wuhan, China

${ }^{2}$ Department of Cardiovascular Surgery, Zhongnan Hospital of Wuhan University, Wuhan, China

${ }^{3}$ Department of Internal Medicine \& Geriatrics, Zhongnan Hospital of Wuhan University, Wuhan, China

${ }^{4}$ Department of Critical Care Medicine, Zhongnan Hospital of Wuhan University, Wuhan, China

${ }^{5}$ Division of Cardiac Surgery, The Ohio State University Wexner Medical Center, Columbus, OH 43210, USA.

${ }^{6}$ Department of Gastroenterology, Zhongnan Hospital of Wuhan University, Wuhan, China

${ }^{7}$ Department of Pathology, Zhongnan Hospital of Wuhan University, Wuhan, China

${ }^{*}$ Co-corresponding author

Xuefeng Zhou: snowingpeak@sina.com

Sufang Tian: usa2005tiansufang@,hotmail.com

Jun Lin: linjun64@126.com

Funding This project was supported by the Program of Excellent Doctoral (Postdoctoral) of Zhongnan Hospital of Wuhan University (Grant No. ZNYB2019002). 


\section{Abstract: \\ BACKGROUND}

The Corona Virus Disease 2019 (COVID-19) is spreading globally now. However, the clinical presentation that predict prognosis of the patients are still largely unknow.

\section{METHODS}

We enrolled 393 patients infected with COVID-19 and 30 patients with common pulmonary bulla and reviewed their clinical features to evaluate the potential prognostic value of pulmonary vesicles, especially in the patients with severe symptoms. One COVID-19 patient with vesicles was treated by bullectomy for last resort, and its characteristics of the patient's perioperative laboratory tests was analyzed. The pathological findings of bullectomy were described and compared with those of common bulla cases.

\section{RESULTS}

Patients infected with COVID-19 showed more dependence on ventilator, occurrence of super resistant bacteria, and prone to vesicle formation than common bulla $(p<0.05)$. Disease severity is associated with age, sex, and usage of ventilator, ECMO and antibiotics, super resistance bacteria and vesicle formation $(p<0.05)$. The average mortality rate of COVID-19 patients was $4.10 \%$ (25.4\% in severe patients, $0.00 \%$ in mild patients). Interestingly, the mortality rate further increased in severe patients with pulmonary vesicles than those without pulmonary vesicles $(35.7 \%$ vs $22.4 \%$, $p=0.0442)$. One COVID-19 patient with vesicles underwent bullectomy and had a poor prognosis, who showed diffuse alveolar damage and extensive necrosis in bullectomy specimen.

\section{CONCLUSIONS}

Patients infected with COVID-19 are more prone to form pulmonary vesicles showed on chest CT scans, as an important poor prognosis factor, especially in the severe patients.

Key words: Corona Virus Disease 2019; pulmonary vesicle; prognosis; surgical treatment 


\section{Introduction}

Pulmonary bullae are cavities of more than $1 \mathrm{~cm}$ in the lung, formed from structurally damaged lung tissue due to a variety of etiologies ${ }^{1}$. When nonoperative approaches for pulmonary bullae turn out to be ineffective, in select cases, surgical resection can be considered ${ }^{2,3}$. Acquired vesicles formed from a local obstructive process due to the formation of a valve which results in bronchiolitis and edema, and as the distal alveolar pressure increases, a vesicle can form and rupture ${ }^{4}$.

The Corona Virus Disease 2019 (COVID-19) pandemic presents with a severe phenotype in as many as $26 \%$ of patients. Medical therapy is lacking, and many of these patients require intubation and even extracorporeal life support (ECLS) ${ }^{5,6,7}$. COVID-19 mainly attacks the lung and also other organs that express ACE2 receptor ${ }^{8}$ 9. Patients with pneumonia infected COVID-19 have been reported to develop pulmonary vesicles and tension pneumothorax with the usage of ventilator. For the pulmonary vesicles, we define it as peripheral predominant consolidation pattern with a round cystic change internally ${ }^{10}$. Because of the high-risk of healthcare worker transmission and the difficulty in performing an operation with full personal protective equipment (PPE), operations on patients carrying COVID-19 are extremely difficult, thus, they are extremely rare. Recently, we performed bullectomy under special circumstances as the last resort. This research compares the COVID-19 patients with pulmonary vesicles and common pulmonary bulla in the clinical data, analyzes the characteristic of pulmonary vesicles after infection of COVID-19, and provides lungrelated surgical experiences for COVID-19 patients with pulmonary vesicles in the future.

\section{Methods}

\section{Study design and patients}

We collected 393 patients infected with COVID-19 and 30 patients with common pulmonary bulla (without COVID-19) admitted to Zhongnan Hospital of Wuhan University from Jan 1, 2019 to Mar 20, 2020 and differences of clinical characteristics between the two groups were analyzed. COVID-19 patients included 63 severe patients (treated in ICU) and 351 mild patients (treated in common ward) based on their clinical data and the New Coronavirus Pneumonia Prevention and Control Program (4th edition) published by the National Health Commission of China ${ }^{11}$. We analyzed the relationship between vesicles and disease severity and the severe patients' neutrophils, lymphocytes, and prognosis. One COVID-19 patient with vesicles (combined with severe ARDS, ECMO support) underwent bullectomy. In order to further study the effect of COVID19 infection on pulmonary vesicles, we performed histochemical analysis with the tissue sections of the patient who underwent surgery, to explore the potential events that may occur when COVID-19 attacks lung tissues.

Diagnosis of COVID-19 pneumonia was based on the New Coronavirus Pneumonia Prevention and Control Program (4th edition) published by the National 
Health Commission of China ${ }^{11}$. All patients infected with COVID-19 were tested positive for severe acute respiratory syndrome coronavirus 2 (SARS-CoV-2) by use of quantitative RT-PCR (qRT-PCR) on samples from the respiratory tract. This study was reviewed and approved by the Medical Ethical Committee of Zhongnan Hospital of Wuhan University (approval number 2020077). Written informed consent was obtained from each enrolled patient.

\section{Data collection}

We reviewed clinical records, laboratory findings, and chest CT scans for the patients. All information was obtained and curated with a customised data collection form. Two study investigators (HT and LZ) independently reviewed the data collection forms to verify data accuracy.

Positive confirmatory cases of COVID-19 infection were defined by clinical laboratory at Zhongnan Hospital of Wuhan University. The clinical data of all patients were collected from the Hospital Information System (HIS), Zhongnan Hospital. Patients infected with COVID were tested for viral nucleic acid through throat swabs, and blood samples were tested for nucleic acid antibodies. The pulmonary sample was obtained from one COVID-19 patient with pulmonary vesicles who underwent emergent bullectomy for persistent pneumothorax and air leak. All perioperative data of this patient was obtained from the HIS, and the intraoperative photos and videos were obtained by an iPhone 8 mobile phone. The pathological staining and diagnosis of this patient were performed by the Department of Pathology, Zhongnan Hospital of Wuhan University.

\section{Histology and immunohistochemistry}

The bullectomy specimen obtained from the right upper lobe of the lung was processed according to routine biosafety standards. Hematoxylin and eosin (H\&E) stained sections were prepared. Immunohistochemical stains were performed using antibodies against $\mathrm{T}$ cell markers such as CD3, CD4 and CD8, and histiocytic marker CD68 (LEICA BIOSYSTEMS, China). All antibodies were in prediluted form and IHC was performed using an autostainer (Leica Bond Max). Special stains such as Gram, periodic acid-Schiff (PAS) and acid-fast bacillus (AFB) stains for microorganisms as well as histochemical stains including elastin and Masson trichrome stains were done on representative tissue blocks.

\section{Statistical analysis}

Statistical analysis was done with SPSS, version 22.0. Independent sample T test were used for statistical analysis between groups. Proportions for categorical variables were compared using the $\chi 2$ test, although the Fisher exact test was used when the data were limited. For unadjusted comparisons, a 2 -sided $\alpha$ of less than .05 was considered statistically significant. The analyses have not been adjusted for multiple comparisons and, given the type I error, the findings should be interpreted as exploratory and descriptive. We used Kaplan-Meier curves to compare patient survival in groups.

\section{Results}


The cases were divided into groups, COVID-19 patients and patients with common bulla. The COVID-19 patients were then grouped into severe patients (treated in ICU) and mild patients (treated in common ward), based on their clinical data and the New Coronavirus Pneumonia Prevention and Control Program (4th edition) published by the National Health Commission of China ${ }^{11}$. As compared to patients with common bulla, patients infected with COVID-19 (more in female) showed more use of ventilator, more prone to vesicle formation ( $p<0.05$, Table). Severe patients (more in male) exhibited older in age, more use of ventilator, ECMO and antibiotics, and more prone to super resistance bacteria infection $(\mathrm{p}<0.05$, Table) than mild patients. The mortality rate of all patients infected with COVID-19 was $4.10 \%(25.4 \%$ in severe patients, $0.00 \%$ in mild patients). In addition, severe patients were more likely to have pulmonary vesicles than mild patients $(p<0.01)$. Furthermore, the formation of pulmonary vesicles was highly correlated with mortality in severe patients $(35.7 \%$ with pulmonary vesicles vs $22.4 \%$ without pulmonary vesicles, $p=0.0442$, Table, Figure 1 .). Severe patients with pulmonary vesicles also had more neutrophils and fewer lymphocytes (Figure 1.). When analyzing chest CT scans, we found that severe patients infected with COVID-19 were more prone to pulmonary vesicle formation (table and Figure 2) than mild patients and patients with common bulla. We found that COVID19 patient with pulmonary vesicles who underwent bullectomy showed higher rate of infections, fewer platelets, more neutrophils and fewer lymphocytes than normal range (Figure 3. A). The COVID-19 patients with pulmonary vesicles showed varying degrees of liver and kidney damage, mild body temperature elevation, and large changes in fluid intake and output (Figure 3. C). However, the COVID-19 patients with pulmonary vesicles are prone to formation of small vacuoles in the lungs (Although this small vacuole may not eventually develop into a pneumothorax, its formation is irreversible, Figure 3. After the COVID-19 patient underwent surgery, the T lymphocyte and inhibition/cytotoxic T lymphocytes in the patient's circulating blood showed a "W-shaped" curve, natural killer cells and B lymphocyte showed an "Mshaped" curve, helper/inducible T lymphocytes showed a trend of gradual increase, and cytokines (IL-2, 4, 6, 10, Interferon $\gamma$, TNF $\alpha$ ) showed an upward trend as a whole (Figure 3. B). The level of circulating estrogen was higher than the normal range, while the level of ACE was within the normal range (Figure 3. C). The patient had pneumothorax (Figure 3. E) before bullectomy surgery due to the previous alveolar structure (Figure 3. D), with the continuous increase in air cavity pressure and rupture of the air cavity wall (Figure 3. G), which was very different from the normal pulmonary bulla (Figure 3. H). During the operation, we found that the structure of the vesicles is clearly separated from the surrounding normal lung tissue, with very thick wall and poor texture of the surrounding lung tissue (Figure 3. G). As a result, the patient's lung still could not open after surgery (Figure 3. F).

\section{Pathological findings}

The resected lung tissue from the COVID-19 patient measured $6.5 \times 2.3 \times 0.8 \mathrm{~cm}$. A cystic bulla was identified beneath the pleura. The inner wall of the bulla was dark brown with attached mucus. No normal-appearing lung tissue was identified grossly. 
The entire tissue was submitted for histological examination, which showed extensive necrosis and hemorrhage at low power view. The alveolar architecture was still maintained with thickened alveolar walls in some areas but destruction in other areas. There was diffuse alveolar damage, sloughing of epithelial cells and basement membrane denudation. Thick hyaline membranes and small foci of organization were still evident in the airspaces (Figure 4. A), providing a clue of COVID-19 infection. Mucinous exudates could be identified (Figure 4. B). Acute bronchopneumonia was shown, as evidenced by the presence of heavy inflammatory cells consisting of mainly neutrophils with scattered macrophages and lymphocytes (Figure 4. C). Immunohistochemistry confirmed lymphocytopenia in the lung tissue, which were CD4+ $\mathrm{T}$ cell predominant (data not shown). Focal dilated airspaces and fibrosis alternating with collapsed alveoli were observed, consistent with bulla formation (Figure 4. D). There were diffuse thickening of alveolar walls and interstitial fibrosis adjacent to the pleura. Vascular congestion, thrombi, and fibrinoid necrosis of blood vessels were noted. There was suspected positive staining for Gram while PAS and AFB stains were negative for fungal and mycobacterial organisms.

In comparison, 4 control cases without COVID-19 infection displayed typical histological features of emphysematous bullae of the lung. Some areas showed loss of alveolar walls due to emphysema and dilation of remaining airspaces. Other areas exhibited prominent interstitial fibroblast hyperplasia and fibrosis (Figure 4. E, F). As for inflammatory infiltrates, there was an increased number of lymphocytes close to bronchioles, small vessels and along the pleura. Macrophages were noted in the airspaces. No obvious neutrophils could be identified (Figure 4. G, H). The ratio of CD4+ to CD8+ T cells was normal (data not shown). On elastin staining, the COVID19 case showed fine elastin fiber deposition, which was difficult to appreciate on H\&E stain because of extensive necrosis (Figure 4. I). Thickened elastic fibers could be identified but less obvious than those seen in control cases (Figure 4. J).

\section{Discussion}

The etiology of pulmonary bulla is complex, including chronic obstructive pulmonary disease (COPD), emphysema, ventilator-related lung injury, and now, COVID-19 1, 12. The pulmonary vesicles showed on the chest CT scans, peripheral predominant consolidation pattern with a round cystic change internally ${ }^{10}$, are more prone to pulmonary bullous formation. However, the influence of COVID-19 on pulmonary vesicles has not been reported. Our study found that patchy ground-glass shadow appeared on chest CT scans in the early stage of COVID-19 infection, which is consistent with a previous report ${ }^{10}$. As disease progresses, the patchy shadows gradually expand, with vesicles gradually forming at the origin area of the shadow. The formation of the vesicles was due to alveolar structure destruction which led to alveolar fusion. As alveolar structure destruction occurred quickly, the wall of air cavity was significantly thicker than that of common pulmonary bulla (Figure 3. G, H). In this process, the inflammatory storm was also an important factor ${ }^{5}$. Thus, we believe that 
the destruction of alveolar structure due to COVID-19 is easy to induce emphysema, and then causes the pulmonary vesicles.

In addition to the etiology, we want to further explore the potential prognostic value of small pulmonary vesicles for COVID-19 patients. For the COVID-19 severe patients with vesicles, the mortality rate increased to $35.7 \%$ from $22.4 \%$ of the severe patients without vesicles, thus pulmonary vesicles are very important poor prognostic factor to predict the mortality of the patients. Furthermore, the use of antibiotics led to the emergence of multi-drug resistant bacteria and further deterioration of lung oxygenation, eventually relying more on the ventilator. And then, mechanical ventilation made the alveolar destruction further aggravated, with vicious cycle. So, we speculate the most reasonable mechanism including the directly injury from COVID19 , excessive breath drive in spontaneous breathing, manifested as too fast respiratory rate $(>30 \mathrm{bpm})$ and excessive chest negative pressure, and the ventilator induced lung injury.

Although pulmonary vesicles are not the true bulla, they easily develop into bullae. The most effective approach to treat symptomatic pulmonary bulla is surgical resection ${ }^{13}$, which is widely accepted by thoracic surgeons all over the world. Nevertheless, it was a difficult choice for COVID-19 patients with pulmonary vesicles. It is clear that when patients have tension pneumothorax, chest drainage tube must be performed as soon as possible. Most COVID-19 patients with poor oxygen saturation and severe ARDS required ventilatory support because of the severe damage to the lung tissue from COVID-19 infection ${ }^{8,14}$. In this study, one patient underwent bullectomy, with pulmonary vesicles induced by COVID-19 and tension pneumothorax.

To our knowledge, this operation was the first bullectomy performed on COVID19 patient with both gross and histologic findings. We had to resect the pulmonary vesicles tissue for the patient. During the operation, we found that the quality of lung tissue was extremely poor. But the cutting edge of the lung tissue still had discontinuous air leakage after the resection, and air leakage was still found at the new cutting edge even repaired the cutting edge. So, the operation was completed with $90 \%$ satisfaction. The lung tissues infected with COVID-19 are often combined with a variety of microbial infections ${ }^{15}$, leading to a severe local inflammatory response and tissue destruction. Combined with preoperative CT, operation and postoperative clinical analysis, we further confirmed the inflammatory storm of local inflammatory reactions in the lungs as reported before ${ }^{5}$. Regrettably, the outcome of this patient is poor after bullectomy (Figure 3. F).

This is also the first report on pathological findings of COVID-19 complicated by emphysematous bulla formation in the lung. We have reported pulmonary pathology on early phase of COVID-19 in two cases undergoing lobectomies for cancer ${ }^{16}$ and more advanced changes in four cases through postmortem core biopsies. The present case extended the morphology spectrum of COVID-19. Although extensive degeneration and necrosis present, hyaline membranes and early organization could still be identified, which indicated subacute phase of diffuse alveolar damage. The distribution and subsets of lymphocytes were consistent with his CBC profile, which was lymphocytopenia. The relationship between pathogenesis and the microscopic 
changes in the present case is not clear. A number of potential mechanisms may be involved based on literature review and our own clinical observations. It is known that the pathogenesis of bulla formation involves but is not limited to inflammatory mediators and leukocytes, proteases and anti-protease imbalance, oxidative stress and infection 17. Specifically, proteases such as elastase and extracellular matrix metalloproteinase-1 and -9 , cytokines and chemokines usually contribute to the formation of emphysematous bullae and pneumothorax ${ }^{18,19}$. It was suggested matrix metalloproteinase-9 was predominantly overexpressed in neutrophils, alveolar macrophages, and mesothelial cells ${ }^{18}$. Interactions of multiple factors, including diffuse alveolar damage overlapped with extensive necrosis, abundant neutrophils in lung tissue which can produce matrix metalloproteinase, combined with elevated levels of cytokines such as interleukins in the peripheral blood, may eventually lead to bulla formation in our case.

In conclusion, COVID-19 may induce the pulmonary vesicles showed on the chest CT scans, which has a thicker air cavity wall than common bulla. Considering ventilator-related lung injury, it is recommended to choose the ventilator mode and PEEP carefully. Patients infected COVID-19 are more prone to form pulmonary vesicles which were easy evaluated by chest CT timely and objectively, as an important poor prognosis factor with high mortality, especially in the severe patients. Base on the extensive destruction of lung tissue by COVID-19, bullectomy is limited, only as a last resort and trial treatment if the patient accepted. More research is needed to explore the specific mechanisms of formation of pulmonary vesicles to improve the efficacy of COVID-19 pneumonia treatment, especially in severe COVID-19 patients with vesicles.

\section{Contributors}

HT, LZ, and YW conceived and designed the study. YW and CL contributed to the literature search and data collection. ST contributed to pathology examination and interpretation of the findings. HT, LZ, BH, XZ, and JL contributed to data interpretation. $\mathrm{HX}, \mathrm{LZ}, \mathrm{NAM}, \mathrm{HZ}$, and $\mathrm{XZ}$ contributed to the figures and writing of the report.

\section{Declaration of interests}

NAM is consultant to Medtronic, Abbott, SynCardia, Carmat.

\section{Acknowledgments}

We thank all our colleagues who helped us during the current study. We greatly appreciate the kind assistance of Professor Bicheng Wang and Doctor Lijun Cai (Both are in the Department of Pathology, Zhongnan Hospital of Wuhan University, Wuhan, China) in staining the sections, as well as Doctor Xianguo Wang and Doctor Zetian Yang (Both are in the Department of Thoracic Surgery, Zhongnan Hospital of Wuhan University, Wuhan, China) for his assistance in discussing the key issues of this study. We are also grateful to the many frontline medical staff for their dedication in the face of the COVID-19 outbreak, despite the potential threat to their own lives and the lives of their families. 


\section{REFERENCES}

1. Erne BV, Graff M, Klemm W, Danzl JG, Leschber G. Bulla in the lung. Lancet 380, 1280 (2012).

2. Ozawa Y, Sakai M, Ichimura H. Covering the staple line with polyglycolic acid sheet versus oxidized regenerated cellulose mesh after thoracoscopic bullectomy for primary spontaneous pneumothorax. General thoracic and cardiovascular surgery 66, 419-424 (2018).

3. Sim SKR, Nah SA, Loh AHP, Ong LY, Chen Y. Mechanical versus Chemical Pleurodesis after Bullectomy for Primary Spontaneous Pneumothorax: A Systemic Review and MetaAnalysis. European journal of pediatric surgery : official journal of Austrian Association of Pediatric Surgery [et al] = Zeitschrift fur Kinderchirurgie, (2019).

4. Byrd RP, Jr., Roy TM. Spontaneous resolution of a giant pulmonary bulla: what is the role of bronchodilator and anti-inflammatory therapy? Tennessee medicine : journal of the Tennessee Medical Association 106, 39-42 (2013).

5. Wang D, et al. Clinical Characteristics of 138 Hospitalized Patients With 2019 Novel Coronavirus-Infected Pneumonia in Wuhan, China. JAMA, (2020).

6. Yang W, et al. Clinical characteristics and imaging manifestations of the 2019 novel coronavirus disease (COVID-19):A multi-center study in Wenzhou city, Zhejiang, China. The Journal of infection, (2020).

7. Guan WJ, et al. Clinical Characteristics of Coronavirus Disease 2019 in China. N Eng/ J Med, (2020).

8. Zhou F, et al. Clinical course and risk factors for mortality of adult inpatients with COVID19 in Wuhan, China: a retrospective cohort study. Lancet, (2020).

9. Zhou P, et al. A pneumonia outbreak associated with a new coronavirus of probable bat origin. Nature 579, 270-273 (2020).

10. Shi $\mathrm{H}$, et al. Radiological findings from 81 patients with COVID-19 pneumonia in Wuhan, China: a descriptive study. The Lancet Infectious diseases, (2020).

11. Chen $\mathrm{H}$, et al. Clinical characteristics and intrauterine vertical transmission potential of COVID-19 infection in nine pregnant women: a retrospective review of medical records. Lancet 395, 809-815 (2020).

12. Stanescu D, Veriter CL. Spontaneous regression of a giant pulmonary bulla. Thorax 51, 1283 (1996). 
13. Neidhart P, Suter PM. Pulmonary bulla and sudden death in a young aeroplane passenger. Intensive Care Med 11, 45-47 (1985).

14. Rubin EJ, Baden LR, Morrissey S. Audio Interview: What Clinicians Need to Know in Diagnosing and Treating Covid-19. N Eng/ J Med 382, e19 (2020).

15. Qin C, et al. Dysregulation of immune response in patients with COVID-19 in Wuhan, China. Clinical infectious diseases : an official publication of the Infectious Diseases Society of America, (2020).

16. Tian S, Hu W, Niu L, Liu H, Xu H, Xiao SY. Pulmonary Pathology of Early-Phase 2019 Novel Coronavirus (COVID-19) Pneumonia in Two Patients With Lung Cancer. J Thorac Oncol, (2020).

17. Kumar V, Abbas AK, Aster JC. Robbins and Cotran pathologic basis of disease, Ninth edition. edn.

18. Chiu CY, et al. Overexpression of matrix metalloproteinase-9 in adolescents with primary spontaneous pneumothorax for surgical intervention. J Thorac Cardiovasc Surg 156, 2328-2336 e2322 (2018).

19. Hayes JA, Korthy A, Snider GL. The pathology of elastase-induced panacinar emphysema in hamsters. J Patho/ 117, 1-14 (1975). 
Table. Clinical characteristics of the patients

\begin{tabular}{|c|c|c|c|c|c|c|}
\hline Group & $\mathrm{A} 1(n=63)$ & A2 $(n=330)$ & $\boldsymbol{P}$ & $A(n=393)$ & $B(n=30)$ & $\boldsymbol{P}$ \\
\hline Age(vears) & $61(21,87)$ & $56(13,93)$ & $0.010^{*}$ & $57(13,93)$ & $59(21,83)$ & 0.392 \\
\hline \multicolumn{7}{|l|}{ Sex } \\
\hline Male. n (\%) & $41(65.10)$ & $147(44.50)$ & \multirow{2}{*}{$0.003^{*}$} & $188(47.80)$ & $26(86.70)$ & \multirow{2}{*}{$<0.01^{*}$} \\
\hline Female. n (\%) & $22(34.90)$ & $183(55.50)$ & & $205(52.20)$ & $4(13.30)$ & \\
\hline \multicolumn{7}{|l|}{ DH } \\
\hline Asthma. n (\%) & $9(14.29)$ & $40(12.10)$ & 0.634 & $49(12.50)$ & $3(10.00)$ & 0.691 \\
\hline COPD. $\mathrm{n}(\%)$ & $12(19.05)$ & $38(11.50)$ & 0.100 & $50(12.70)$ & $2(6.67)$ & 0.330 \\
\hline \multicolumn{7}{|l|}{ Treatment } \\
\hline No-S. n $(\%)$ & $58(92.10)$ & $330(100)$ & \multirow{2}{*}{$<0.01^{*}$} & $388(98.70)$ & 0 & \multirow{2}{*}{$<0.01^{*}$} \\
\hline S.n $(\%)$ & $5(7.90)$ & 0 & & $5(1.30)$ & $30(100)$ & \\
\hline \multicolumn{7}{|l|}{ Life support } \\
\hline V.n $(\%)$ & $28(44.44)$ & 0 & & $28(7.12)$ & 0 & \\
\hline IV.n $(\%)$ & $19(67.86)$ & 0 & & $19(67.86)$ & 0 & \\
\hline Non-IV. n (\%) & $9(32.14)$ & 0 & & $9(32.14)$ & 0 & \\
\hline ECMO. n $(\%)$ & $8(12.70)$ & 0 & & $8(2.04)$ & 0 & \\
\hline \multicolumn{7}{|l|}{ Antimicrobial } \\
\hline Antibiotics. n (\%) & $63(100)$ & $132(40.00)$ & $<0.01^{*}$ & $195(49.60)$ & $30(100)$ & $<0.01^{*}$ \\
\hline LGA. n (\%) & $10(15.90)$ & $92(69.70)$ & \multirow{2}{*}{$<0.01^{*}$} & $102(52.30)$ & $26(86.70)$ & \multirow{2}{*}{$<0.01^{*}$} \\
\hline HGA. n (\%) & $53(84.10)$ & $40(30.30)$ & & $93(47.70)$ & $4(13.30)$ & \\
\hline Anti-virus. n (\%) & $63(100)$ & 294(89.10) & $<0.01^{*}$ & $357(90.80)$ & $1(3.33)$ & $<0.01^{*}$ \\
\hline \multicolumn{7}{|l|}{ Complications } \\
\hline SRB. n (\%) & $24(38.10)$ & $35(10.60)$ & $<0.01^{*}$ & $59(15.00)$ & $2(6.70)$ & 0.221 \\
\hline V-related. n (\%) & $13(54.20)$ & $20(57.10)$ & \multirow{2}{*}{0.821} & $33(55.90)$ & $1(50 \%)$ & \multirow{2}{*}{0.872} \\
\hline Blood-related. n (\%) & $11(45.80)$ & $15(42.90)$ & & $26(44.10)$ & $1(50 \%)$ & \\
\hline PV.n $(\%)$ & $14(22.20)$ & $23(7.00)$ & $<0.01^{*}$ & $37(9.41)$ & $30(100)$ & $<0.01^{*}$ \\
\hline \multicolumn{7}{|l|}{ Prognosis } \\
\hline Cure. $\mathrm{n}(\%)$ & $47(74.60)$ & $330(100)$ & \multirow{2}{*}{$<0.01^{*}$} & $377(95.90)$ & $30(100)$ & \multirow{2}{*}{0.260} \\
\hline Dead. n $(\%)$ & $16(25.40)$ & 0 & & $16(4.10)$ & 0 & \\
\hline
\end{tabular}

Abbreviation A1, Severe (Patients treated in ICU); A2, Mild (treated in common ward); A, All Patients infected COVID-19; B, Patients with common bulla; S, Surgery (bullectomy and Chest drainage tube); V, Ventilator; BU, Bullectomy; DH, Disease history; IV, Invasive ventilator; PV, pulmonary vesicles; LGA, Low grade antibiotic; HGA, Hight grade antibiotic; SRB, Super resistant bacteria; COPD, Chronic obstructive pulmonary disease; ECMO, Extracorporeal Membrane Oxygenation. Evaluation of A1, A2, A and B based on the New Coronavirus Pneumonia Prevention and Control Program (4th edition) published by the National Health Commission of China ${ }^{11}$ 
Figure legends

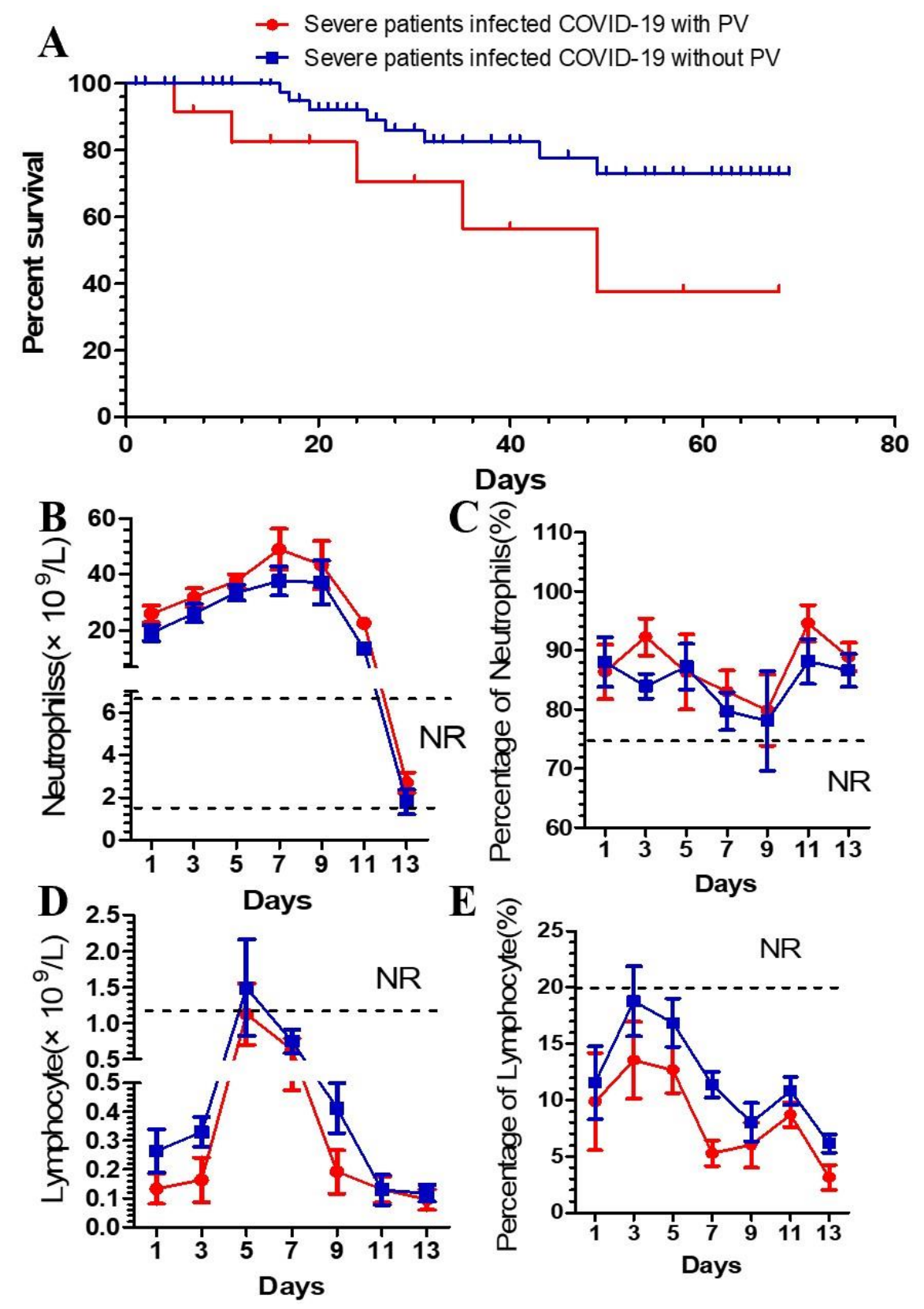

Figure 1. The difference of pulmonary vesicles and without vesicles in severe patients. For the 63 severe patients, 5 of 14 with vesicles dead (35.7\%), and 11 of 49 without vesicles dead $(22.4 \%)$. The survival curve of severe patients with vesicles was significantly shortened $(\mathrm{p}=0.0442$, A). Severe patients with vesicles had lower neutrophils (absolute number, $\mathrm{p}=0.0121, \mathbf{B}$; percentage, $\mathrm{p}=0.0047, \mathbf{C}$ ), and lower percentage of lymphocytes $(\mathrm{p}=0.0128, \mathbf{E})$, but there was no statistical difference in absolute lymphocyte numbers $(\mathrm{p}=0.1853$, D). Abbreviation PV, pulmonary vesicles; $\mathrm{NR}$, normal range. 


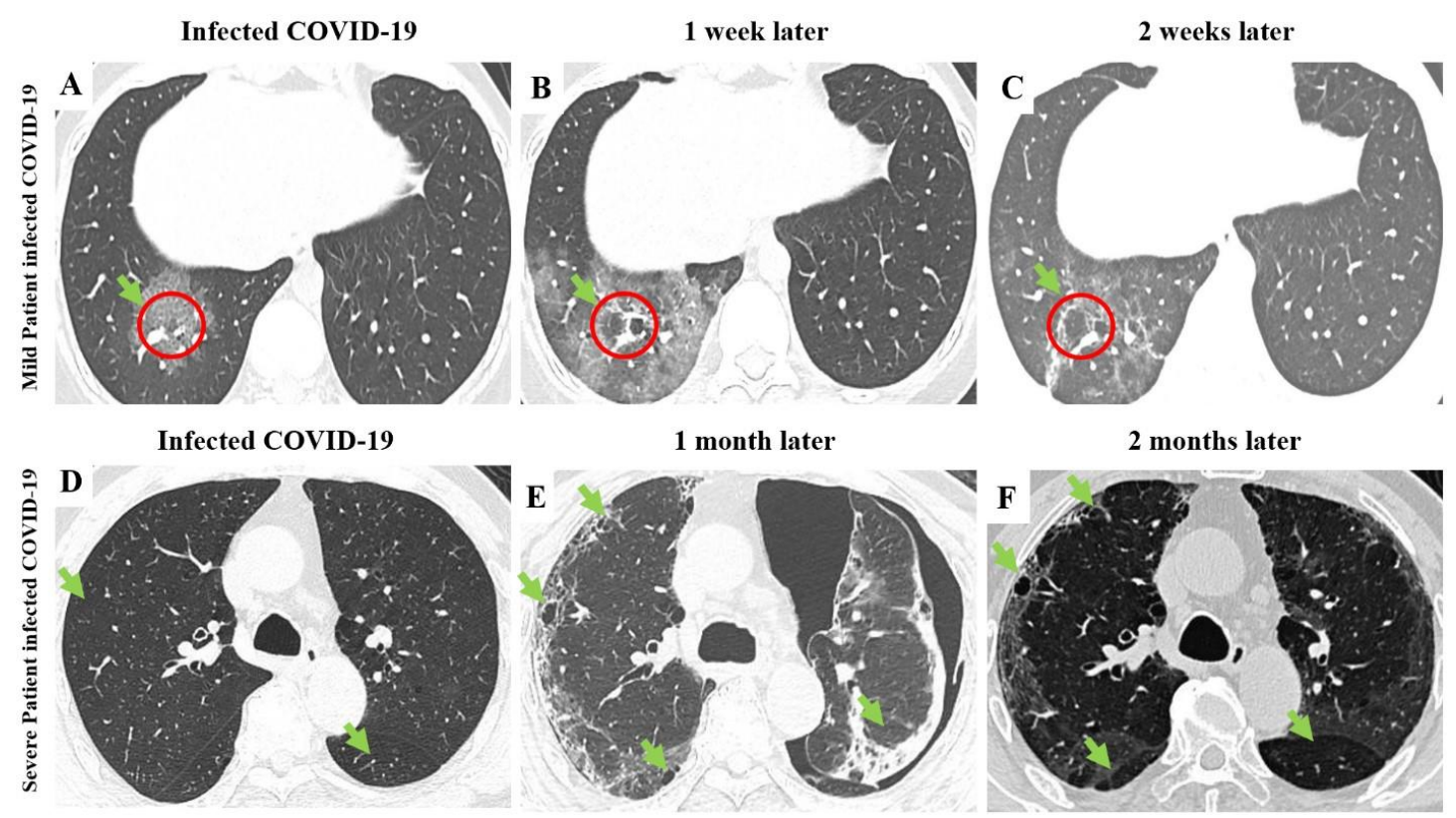

Figure 2. The imaging data of patients. Representative CT images of COVID-19 patients with pulmonary vacuoles were shown. For mild patients (A-C), small vacuoles began to appear after infection and gradually disappeared in the recovery phase ( 1-2 weeks). For the severe patients (D-F), large cavities appeared after infection, and persisted and developed into emphysema after cured. 

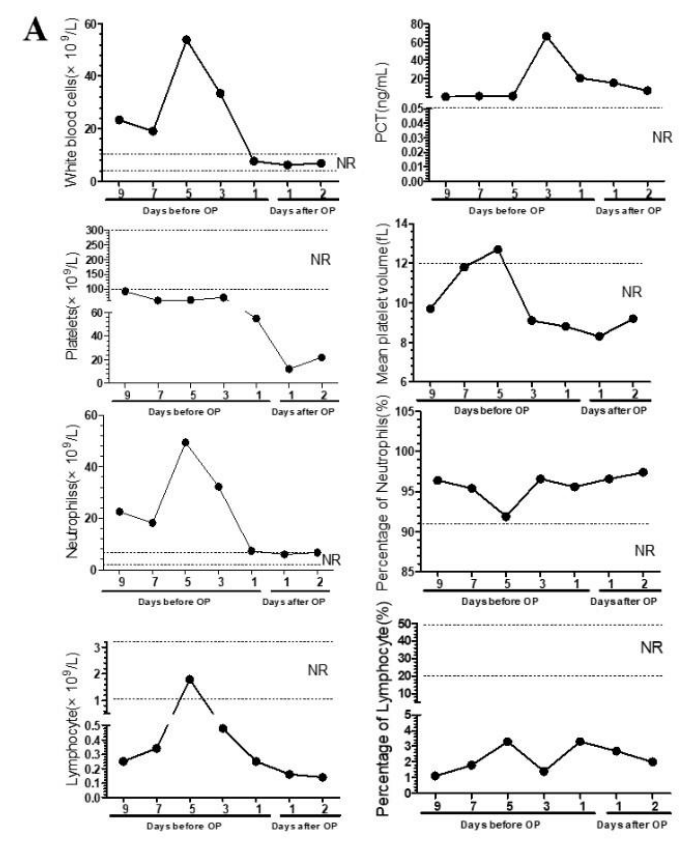

B
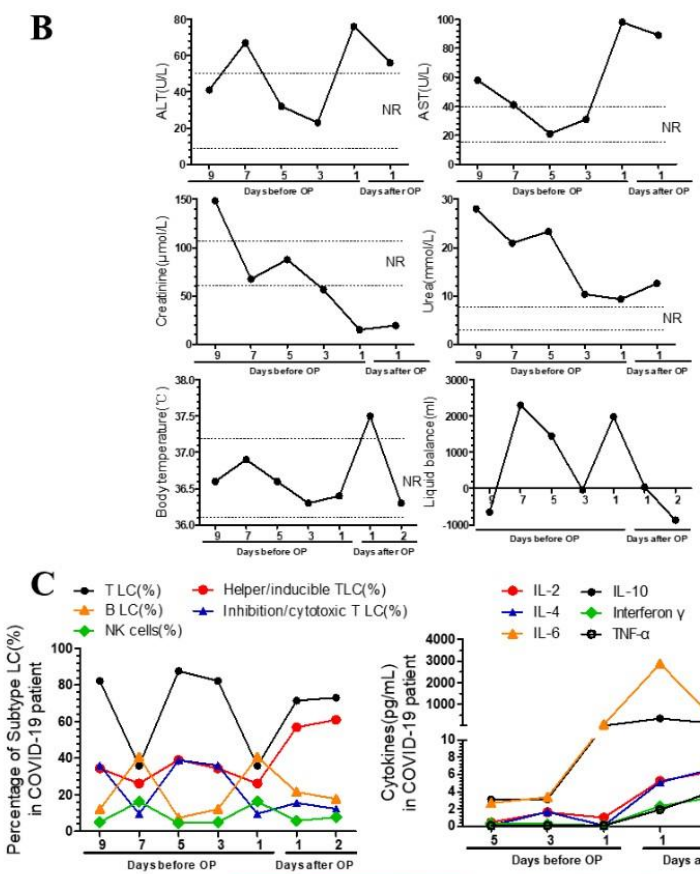

$\rightarrow \mathrm{LL}-2 \rightarrow \mathrm{LL}-10$

$\neq \mathrm{IL}-4 \rightarrow$ Interferon $\mathrm{Y}$
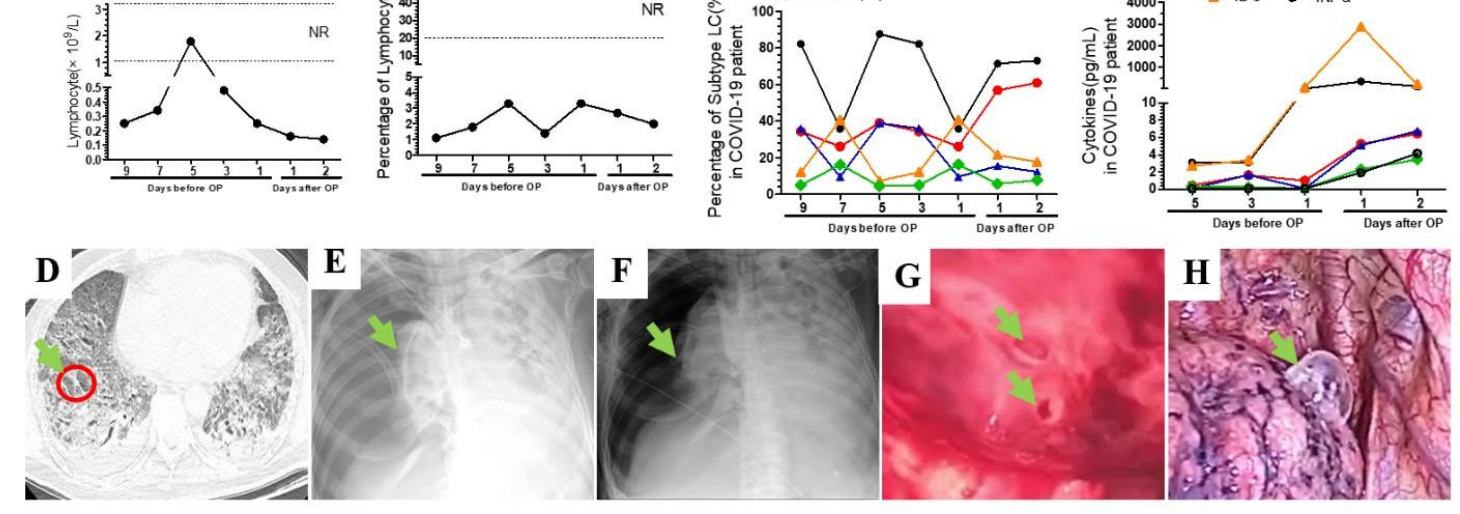

Figure 3. Perioperative laboratory tests on patient underwent surgery. A.

Perioperative blood routine results showed that COVID-19 patient with pulmonary vesicles showed higher rate of infections, fewer platelets, more neutrophils and fewer lymphocytes. B. After receiving surgery, the TLC and I/CLC in the patient's circulating blood showed a "W-shaped" curve, NK cells and BLC showed an "Mshaped" curve, H/I LC showed a trend of increasing gradually, and cytoknes (IL-2, 4, 6,10 , Interferon $\gamma$, TNF $\alpha$ ) showed an upward trend as a whole. C. The level of circulating estrogen was higher than the normal range, while the level of ACE was in the normal range. The COVID-19 patient with pulmonary vesicles showed varying degrees of liver and kidney damage, mild body temperature elevation, and large changes in fluid intake and output. D. 20 days before bullectomy, the chest CT scans of the patient showed changes in small vesicles in the lung. E. Chest X-ray, before bullectomy. F, Chest X-ray, after bullectomy. G. the pulmonary vesicles of the patient during the bullectomy. H. a common pulmonary bulla. Abbreviation NR, normal range; PCT, Procalcitonin; TLC, T lymphocyte; BLC, B lymphocyte; I/CLC, Inhibition/cytotoxic T lymphocytes; NK cells, Natural killer cells; H/I LC, Helper/inducible T lymphocytes. 


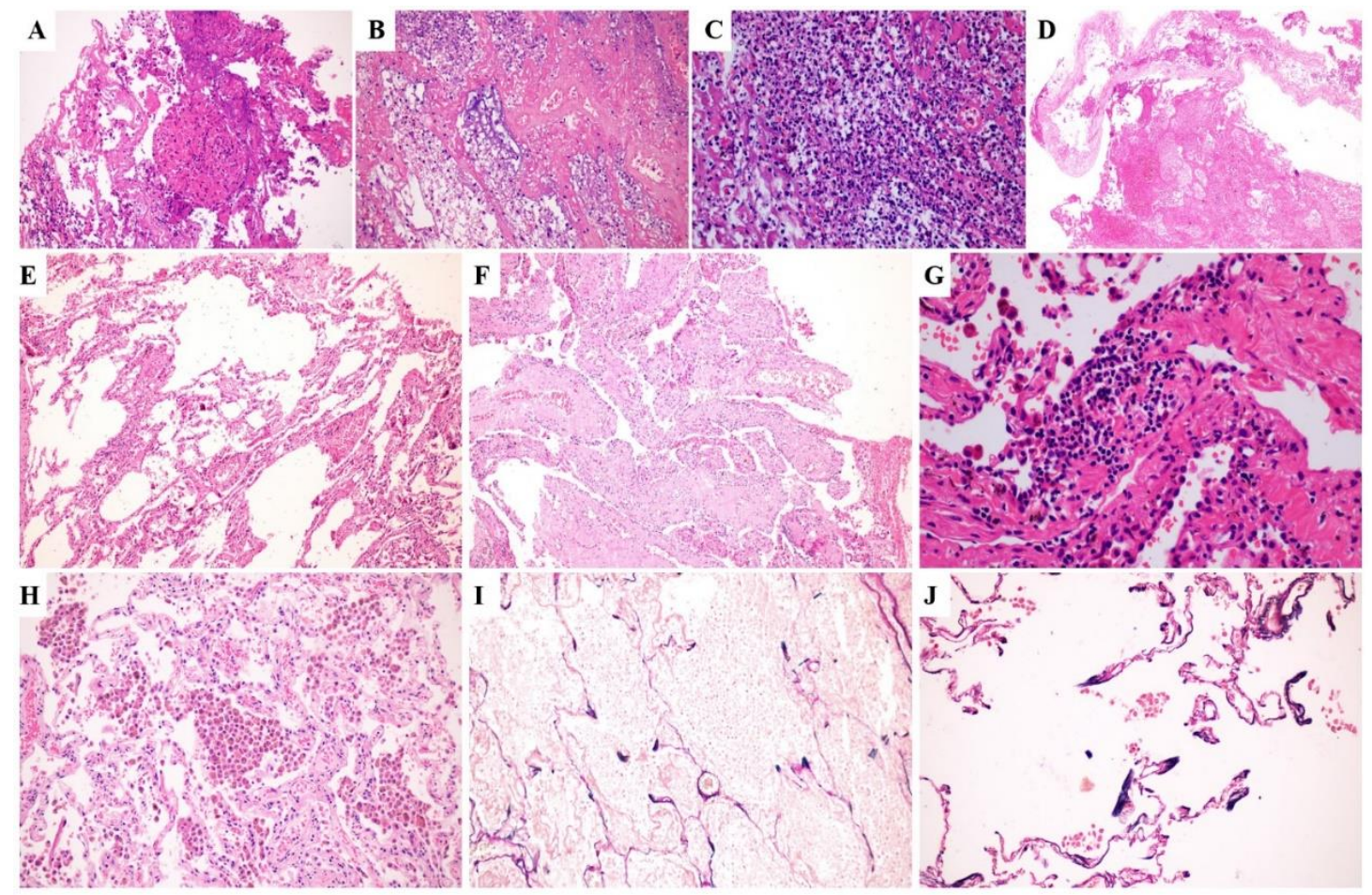

Figure 4. Pathological findings of bullectomies from patients with and without COVID-19. COVID-19 case (A-D): A, Fibrin exudates, hyaline membranes and small foci of organization in airspaces. B, Mucinous exudates. C, Abundant inflammatory cell infiltrates consisting mainly of neutrophils. D, Degenerated vesicles. Non-COVID-19 cases (E-H): E, Loss of alveolar walls and dilation of airspaces due to emphysema. F, Markedly thickened interstitial spaces. G, Patchy increase in the number of lymphocytes. H, Macrophages seen in airspace. Elastin staining on COVID-19 case (I) and non-COVID-19 cases (J). Fine elastic fibers with occasional thickened fibers seen in COVID-19 case (I). Thickened elastic fibers seen in a nonCOVID-19 case (J). 